\title{
Autopsy Findings in Patients with Uterine Sarcoma
}

\author{
William P. Fleming, M.D., ' William A. Peters III, M.D., Neelam B. Kumar, \\ M.D., and George W. Morley, M.D.
}

Department of Obstetrics and Gynecology and Pathology, University of Michigan Hospitals, Ann Arbor, Michigan 48109

Received September 12, 1983

\begin{abstract}
Autopsy findings were reviewed in 22 patients treated for uterine sarcoma at the University of Michigan Hospitals. Included are 11 mixed mesodermal tumors, 6 endometrial stromal sarcomas, and 5 leiomyosarcomas. Only one patient died with disease limited to the pelvis. Forty-five percent of the patients died with disease limited to the pelvis and abdomen. The most common site of disease above the diaphragm was the lung. Lymph nodes were involved in $59 \%$ of our patients. There were no obvious differences in the patterns of spread between the tumor types. (c) 1984 Academic Press, Inc.
\end{abstract}

\section{INTRODUCTION}

Uterine sarcomas represent a rare, but interesting group of tumors that are among the most malignant of gynecological cancers. The biologic behavior of these tumors remains poorly understood and attempts to elucidate their clinical and pathological features have been frustrated by the multiple and often complex classification schemes utilized. Surgical therapy will salvage some patients with limited disease, but the survival rates in patients with extrauterine disease are uniformly poor and there has been no significant advance in adjuvant therapy.

In an effort to improve results it will be necessary to alter treatment protocols by taking into account the biological behavior of these tumors. This report examines the autopsy findings of a group of patients treated for uterine sarcomas by evaluating their pattern of spread and searching for differences between the tumor types which might alter the approach to therapy.

\section{MATERIALS AND METHODS}

A review was made of all patients treated for uterine sarcoma at the University of Michigan Hospitals between January 1, 1936 and June 30, 1983. Those patients having a postmortem examination were identified and are the subject of this report. The autopsy protocols were studied and the original histology slides reviewed by a gynecologic pathologist. The protocols and slides were obtained

1 To whom correspondence should be addressed at Three Crosses Ob-Gyn, 299 E. Montana, Las Cruces, New Mexico 88005. 
in five cases where the examination was performed elsewhere and similarly reviewed. Additional slides were cut when the originals were of poor quality. Tumors were classified using the criteria of Ober [1]. Those neoplasms containing only endometrial stromal cells were diagnosed as endometrial stromal sarcomas only if they had infiltrating borders and if the mitotic activity was greater than 10 mitoses/10 HPFs [2]. Patients with endolymphatic stromal myosis, a tumor with low malignant potential, were excluded from this study. Leiomyosarcomas were diagnosed in smooth muscle tumors with more than 5 mitoses/10 HPFs with $3+$ cellular atypia or more than 10 mitoses/10 HPFs with any degree of atypia [3]. Mixed mesodermal tumors were divided into homologous and heterologous groups.

\section{RESULTS}

For the period January 1, 1936 to June 30, 1983, 203 patients were evaluated at the University of Michigan Hospitals with a diagnosis of uterine sarcoma. Twenty-two of these patients had a postmortem examination. The group included 5 patients with leiomyosarcoma, 6 patients with endometrial stromal sarcoma, and 11 with mixed mesodermal sarcoma. Three of the autopsies have been previously reported [4].

Two patients with mixed mesodermal tumors had received prior pelvic radiation therapy, one for adenocarcinoma of the endometrium 8 years prior to her presentation and one for squamous carcinoma of the cervix 20 years prior to her presentation with sarcoma. Four patients were treated by surgery only, four patients were treated by surgery and chemotherapy, three patients by surgery and radiation therapy, and two patients by all three modalities. Two patients were treated by radiation alone and two patients solely by chemotherapy. Five patients with far advanced disease received only supportive therapy.

The specific site and frequency of metastases at autopsy are shown in Table 1. All 22 patients had disease in the pelvis, but only one patient had disease confined to the pelvis. The remaining 21 patients died with diffuse upper abdominal disease. Extraabdominal disease was found in 12 patients, with the lung being the organ most frequently involved. Of the 12 patients having brain examinations, 2 showed metastatic brain lesions. Nodal disease occurred in $59 \%$ of our patients. Based on the autopsy records, it was not always possible to distinguish involvement of paraortic and pelvic lymph nodes and they are considered together as retroperitoneal nodes. There were no obvious differences in the patterns of spread among the three subclasses of uterine sarcoma. There were no differences noted between the homologous and heterologous mixed mesodermal tumors.

The histologic nature of the metastases were analyzed in those patients who had mixed mesodermal sarcoma. In four patients the metastases were found to be sarcoma only, two patients had metastases of carcinoma only, and in five patients the metastases were found to be both carcinoma and sarcoma.

The direct causes of death are summarized in Table 2. Obstructive renal failure and respiratory failure due to tumor replacement or pneumonia were the most common direct causes of death. The interval between diagnosis and death averaged 9 months (range 1 day to 47 months). More than $75 \%$ of the patients died within 1 year of discovery of their sarcoma. 
TABLE 1

Distribution of Uterine Sarcoma Metastases at the University of Michigan

\begin{tabular}{|c|c|c|c|c|}
\hline & $\begin{array}{l}\text { Leiomyosarcoma } \\
\text { (5) }\end{array}$ & $\begin{array}{c}\text { Mixed } \\
\text { mesodermal } \\
\text { (11) }\end{array}$ & $\begin{array}{l}\text { Endometrial } \\
\text { stromal } \\
\text { (6) }\end{array}$ & $\begin{array}{c}\text { Total } \\
(22)\end{array}$ \\
\hline \multicolumn{5}{|l|}{ Pelvis } \\
\hline Pelvic peritoneum & 5 & 10 & 6 & 21 \\
\hline Vagina & 2 & 7 & 6 & 15 \\
\hline Retroperitoneal nodes & 2 & 7 & 4 & 13 \\
\hline Bladder muscularis & 1 & 2 & 1 & 4 \\
\hline Rectal muscularis & 0 & 2 & 0 & 2 \\
\hline \multicolumn{5}{|l|}{ Upper abdomen } \\
\hline Upper abdominal perito & & & & \\
\hline including diaphragm & 4 & 11 & 6 & 21 \\
\hline Serosa of bowel & 2 & 8 & 6 & 16 \\
\hline Parenchymal liver & 1 & 6 & 2 & 9 \\
\hline Pancreas & 0 & 3 & 1 & 4 \\
\hline Kidney & 1 & 2 & 0 & 3 \\
\hline Spleen & 1 & 1 & 1 & 3 \\
\hline Bowel muscularis & 0 & 2 & 0 & 2 \\
\hline Adrenal & 0 & 0 & 1 & 1 \\
\hline \multicolumn{5}{|l|}{ Extraabdominal } \\
\hline Lung & 1 & 7 & 2 & 10 \\
\hline Esophagus & 1 & 1 & 0 & 2 \\
\hline Breast & 1 & 0 & 0 & 1 \\
\hline Bone & 0 & 1 & 0 & 1 \\
\hline Brain & $1 / 4$ & $1 / 6$ & $0 / 2$ & $2 / 12$ \\
\hline
\end{tabular}

\section{DISCUSSION}

Uterine sarcomas constitute less than $5 \%$ of all uterine cancers and are characterized by an extremely poor survival rate for those patients who have extrauterine disease. Because of their rarity and the confusing array of classification schemes, no consensus exists as to their mode of spread or response to adjuvant therapy. Our study was undertaken to help elucidate the patterns of spread of uterine sarcoma in treatment failures and to determine if the various histologic subgroups have an affinity for spread to certain sites.

TABLE 2

Direct Cause of Death

\begin{tabular}{ll}
\hline Obstructive renal failure & 7 \\
Respiratory failure & 7 \\
Pulmonary embolus & 2 \\
Bowel perforation & 2 \\
Not specified & 4 \\
\hline
\end{tabular}


Table 3 summarizes a review of previously reported autopsy findings. Because of confusing classification systems used in the past, it was not possible to decipher some of the older reports and this review is limited to reports with sufficient detail to classify. It was particularly difficult to obtain autopsy cases of pure endometrial stromal sarcoma since many of the older reports did not distinguish this tumor from endolymphatic stromal myosis.

The previous findings parallel our own and there were no apparent differences in the patterns of tumor spread among the subgroups studied. Nodal involvement has been frequently reported with mixed mesodermal sarcoma and endometrial stromal sarcoma as was seen in our series (65\% nodal involvement). However, nodal involvement with leiomyosarcoma has been an issue of controversy, but was seen in 2 of 5 patients in our series and in 13 of 29 previously reported autopsies, an overall incidence of $44 \%$. Leiomyosarcomas are rarely diagnosed preoperatively, consequently nodal palpation and sampling is less likely to be done intraoperatively. This may explain the low frequency with which nodal disease has been discussed in clinical papers.

Local control was infrequent with all tumor types. Disease occurrence outside the pelvis was found in 21 of $22(95 \%)$ of our patients and in 56 of $58(97 \%)$ of patients with previously reported autopsies. Overall $45 \%$ of the patients had disease limited to the abdomen and pelvis while 55\% had disease above the diaphragm. It is readily apparent that widespread and distant metastases occur early in the course of the disease process and account for the vast majority of treatment failures, and that leiomyosarcoma, mixed mesodermal, and endometrial stromal sarcoma spread by direct extension, lymphatic, and hematogenous routes. It appears that intraperitoneal spread, in a fashion similar to carcinoma of the ovary, is also very common with these neoplasms.

In summary, our findings reinforce the need for meticulous surgical staging which should include peritoneal washings, sampling of regional and paraortic lymph nodes, and biopsies of the omentum, diaphragm, liver, and peritoneum. Isolated pelvic failure is rare and effective systemic chemotherapy offers the most logical approach to improving survival. Adjuvant radiation therapy probably has a role in control of local disease but is unlikely to influence overall survival.

TABLE 3

Autopsy Findings in Previous Reports

\begin{tabular}{lcccc}
\hline & $\begin{array}{c}\text { Pelvis } \\
\text { only }\end{array}$ & $\begin{array}{c}\text { Pclvis and } \\
\text { upper abdomen } \\
\text { only }\end{array}$ & $\begin{array}{c}\text { Abdominal } \\
\text { and distant }\end{array}$ & $\begin{array}{c}\text { Retroperitoneal } \\
\text { nodes }\end{array}$ \\
\hline $\begin{array}{l}\text { Mixed mesodermal } \\
(24)\end{array}$ & 1 & 7 & 16 & 18 \\
$\begin{array}{l}\text { Leiomyosarcoma } \\
(29)\end{array}$ & 1 & 7 & $21^{a}$ & 13 \\
$\begin{array}{c}\text { Endometrial stromal } \\
\text { sarcoma (5) }\end{array}$ & 0 & 4 & 1 & 2 \\
\hline
\end{tabular}

Note. Compiled from Refs. [2, 5-14].

${ }^{a}$ One patient had a distant metastases only. 


\section{REFERENCES}

1. Ober, W. B. Uterine sarcomas: Histogenesis and taxonomy, Ann. N.Y. Acad. Sci. 75, 568 (1959).

2. Norris, H. J., and Taylor, H. B. Mesenchymal tumors of the uterus. A clinical and pathological study of 53 endometrial stromal tumors, Cancer 19, 755 (1966).

3. Kempson, R. L., and Bari, W. Uterine sarcomas. Classification, diagnosis, and prognosis, Hum. Pathol. 1, 331 (1970).

4. Yoonesi, M., and Hart, W. R. Endometrial stromal sarcoma, Cancer 40, 898 (1977).

5. Kardash, T. Sarcoma of the uterus. Report of two cases with generalized metastases, Amer. $J$. Obstet. Gynecol. 56, 566 (1948).

6. Davis, G. H., Howe, J. S., and French, W. G. Leiomyosarcoma of the uterus. Report of 16 cases, 1917 to 1948, Amer. J. Obstet. Gynecol. 56, 1048 (1948).

7. Wilson, L. A., Graham, L., Thornton, W. N., and Nokes, J. N. Mixed mesodermal tumors of the uterus, Amer. J. Obstet. Gynecol. 66, 718 (1953).

8. Sternberg, W. H., Clark, W. H., and Smith, R. C. Malignant mixed mullerian tumor (mixed mesodermal tumor of the uterus). A study of 21 cases, Cancer 7, 704 (1954).

9. Rubin, A. The histogenesis of carcinosarcoma (mixed mesodermal tumor) of the uterus as revealed by tissue culture studies, Amer. J. Obstet. Gynecol. 77, 269 (1959).

10. Futoran, J. M., and Jesurun, H. M. Endometrial stromal sarcoma, Amer. J. Obstet. Gynecol. 84, 1830 (1962).

11. Spiro, R. H., and Koss, L. G. Myosarcoma of the uterus, a clinicophathologic study, Cancer 18, 571 (1965).

12. Taylor, H. B., and Norris, H. J. Mesenchymal tumors of the uterus. IV. Diagnosis and prognosis of leiomyosarcoma, Arch. Pathol. 82, 40 (1966).

13. Bartsch, E. G., O'Leary, J. A., and Moore, J. G. Carcinosarcoma of the uterus, Obstet. Gynecol. 30, 518 (1967).

14. Lotocki, R., Rosenshein, N. B., Grumbine, F., Dillon, M., Parmely, T., and Woodruff, J. D. Mixed mullerian tumors of the uterus: Clinical and pathologic correlations, Int. J. Gynecol. Obstet. 20, 237 (1982). 